\title{
Flg22-induced Ca2+ increases undergo desensitization and resensitization
}

\author{
Yuan Chi ${ }^{1}$, Chao Wang ${ }^{2}$, Mengyun Wang ${ }^{1}$, Di Wan $^{2}$, Feifei Huang ${ }^{3}$, Zhonghao Jiang ${ }^{3}$, \\ Bridget M. Crawford ${ }^{3}$, Tuan Vo-Dinh ${ }^{3}$, Fang Yuan ${ }^{3}$, Feihua $\mathrm{Wu}^{3}$, and Zhen-Ming $\mathrm{Pei}^{3}$ \\ ${ }^{1}$ Zhejiang University \\ ${ }^{2}$ Hangzhou Normal University \\ ${ }^{3}$ Duke University
}

July 7, 2021

\begin{abstract}
The flagellin epitope flg22, a pathogen-associated molecular pattern (PAMP), binds to the receptor-like kinase FLAGELLIN SENSING2 (FLS2), and triggers Ca2+ influx across the plasma membrane (PM). The flg22-induced increases in cytosolic $\mathrm{Ca} 2+$ concentration $([\mathrm{Ca} 2+] \mathrm{i})(\mathrm{FICA})$ play a crucial role in plant innate immunity. It's well established that the receptor FLS2 and the key downstream component, reactive oxygen species (ROS) burst, undergoes sensitivity adaptation after flg22 stimulation, referred to as desensitization and resensitization, to prevent over responses to pathogens. However, whether FICA also mount adaptation mechanisms to ensure appropriate and efficient responses against pathogens remains poorly understood. Here, we carried out detailed analyses of $[\mathrm{Ca} 2+] \mathrm{i}$ increases upon two successive flg22 treatments, recorded and characterized, for the first time, rapid desensitization but slow resensitization of FICA in Arabidopsis thaliana. Pharmacological analyses showed that the rapid desensitization might be synergistically regulated by ligand-induced FLS2 endocytosis as well as the PM depolarization. The recovery of desensitized FICA might require to de novo FLS2 protein synthesis. FICA resensitization appeared significantly slower than FLS2 protein recovery, suggesting additional regulatory mechanisms of other components, such as flg22-related $\mathrm{Ca} 2+$ permeable channels. Taken together, we have carefully defined the FICA sensitivity adaptation, which will facilitate further molecular and genetic dissection of the Ca2+-mediated adaptive mechanisms in PAMP-triggered immunity.
\end{abstract}

\section{Hosted file}

Chi_PCE-21-0254 Article.pdf available at https://authorea.com/users/424217/articles/529349flg22-induced-ca2-increases-undergo-desensitization-and-resensitization

\section{Hosted file}

Chi_PCE-21-0254 Figures.pdf available at https://authorea.com/users/424217/articles/529349flg22-induced-ca2-increases-undergo-desensitization-and-resensitization 